\title{
Low-Altitude and Slow-Speed Small Target Detection Based on Spectrum Zoom Processing
}

\author{
Xuwang Zhang, Songtao Lu $\mathbb{D}^{1},{ }^{2}$ Jinping Sun $\mathbb{D}^{1},{ }^{1}$ and Wei Shangguan ${ }^{3}$ \\ ${ }^{1}$ School of Electronic and Information Engineering, Beihang University, Beijing 100191, China \\ ${ }^{2}$ Department of Electrical and Computer Engineering, Iowa State University, Ames, IA 50011, USA \\ ${ }^{3}$ National Laboratory of Radar Signal Processing, Xidian University, Xian 710071, China \\ Correspondence should be addressed to Jinping Sun; sunjinping@buaa.edu.cn
}

Received 2 December 2017; Accepted 4 April 2018; Published 10 May 2018

Academic Editor: Wanquan Liu

Copyright (C) 2018 Xuwang Zhang et al. This is an open access article distributed under the Creative Commons Attribution License, which permits unrestricted use, distribution, and reproduction in any medium, provided the original work is properly cited.

\begin{abstract}
This paper proposes a spectrum zoom processing based target detection algorithm for detecting the weak echo of low-altitude and slow-speed small (LSS) targets in heavy ground clutter environments, which can be used to retrofit the existing radar systems. With the existing range-Doppler frequency images, the proposed method firstly concatenates the data from the same Doppler frequency slot of different images and then applies the spectrum zoom processing. After performing the clutter suppression, the target detection can be finally implemented. Through the theoretical analysis and real data verification, it is shown that the proposed algorithm can obtain a preferable spectrum zoom result and improve the signal-to-clutter ratio (SCR) with a very low computational load.
\end{abstract}

\section{Introduction}

The existing radar systems are mainly designed to detect the high-speed military targets such as the fighter plane, missile, and armed helicopter. These targets usually fly at a high altitude with a high speed so that it is very easy to separate the target echo and ground/sea clutter in the frequency domain. In this case, the target detection process of radar systems can be simply described as follows: firstly perform the coherent accumulation and then detect the targets with some traditional constant false alarm rate (CFAR) detection algorithms [1-4]. In practice, the coherent accumulation pulse number is small such that the detection result can be updated quickly with a low computational complexity.

In recent years, there are various types of aircraft, such as the paraglider, light helicopter, and rotorcraft emerging quickly in the market. These aircraft usually fly under 1000 meters with a speed lower than $200 \mathrm{~km} / \mathrm{h}$ and a radar cross section (RCS) smaller than $2 \mathrm{~m}^{2}$. Therefore, these aircraft are mostly called the low-altitude and slow-speed small (LSS) targets, and they have the common characteristics: simple manipulation, easy accessibility, and excellent concealment. Nowadays, the LSS target detection and tracking has become an important research direction in the field of radar signal processing and has a great significance in protecting the safety of major events and maintaining the order of airport flights. For a LSS target, the small RCS signifies that the target echo is very weak, and the low flying height usually results in the mixture of the target echo and heavy ground clutter. In the time domain, the ground clutter has a noticeable mask effect on the target echo. Meanwhile, the Doppler frequency of ground clutter is very close to that of target echo when the target speed is slow. Therefore, it is also difficult to separate the target echo and ground clutter in the frequency domain. All these factors make the LSS target detection very challenging. Unfortunately, the existing radar systems cannot be competent for this problem. Consequently, it is necessary to retrofit the existing radar systems such that they can obtain the LSS target detection ability but also keep the high-speed target detection ability.

There have been already many studies in the literatures focusing on the problem of detecting LSS targets under the background of sea clutter. For example, a short-time 
fractional Fourier transform based detection algorithm was proposed in [5] by investigating the micro-Doppler effect of the targets at the sea surface. In order to improve the detection performance, an adaptive waveform was designed dynamically in [6], where the expectation-maximization algorithm is used to estimate the time-varying parameters of the compound-Gaussian sea clutter. For the maneuvering target detection, two algorithms which apply the adaptive Chirplet decomposition and spectral subtraction, respectively, were proposed in [7]. A time-frequency iteration decomposition method was proposed in [8] by focusing on the nonstationarity of scattered echo from the slow moving weak target at the sea surface. Meanwhile, a time-frequency method was also applied to detect the small accelerating target in the background of sea clutter [9]. In addition, FourierBessel transform was combined with time-frequency analysis to decompose the nonstationary echo of the maneuvering target into multiple components [10]. Besides the method of time-frequency analysis applied in maneuvering target detection problems, the effectiveness of track-before-detect (TBD) method in suppressing the sea clutter was assessed with the real data [11]. Furthermore, a polynomial fitting based signal phase training structure was studied in [12] for the LSS target detection in the sea clutter.

In fact, it is also very significant to study the LSS target detection problem under the background of the ground clutter especially in the complex urban environment. With the fast development of the technology and reduction of the price of aircraft, a large number of paragliders and rotorcrafts appear in the people's daily life. These cheap aircraft mainly fly on the ground and the illegal manipulation on these aircraft can result in serious security issues. However, there are few literatures focusing on the slow moving target detection in the ground clutter [13-17]. A space-time adaptive processing method was proposed to detect the ground moving target with range migration (RM) [14]. In order to reduce the amount of echo data and achieve a wide observation swath, a parametric sparse representation method was used for the motion parameter estimation of the ground target in [15]. In particular, some spectrum zoom algorithms provide a new path for the LSS target detection. For example, the chirp-Z transform (CZT) which can be used to obtain an arbitrary frequency resolution was introduced in [18]. Based on the CZT, an interlaced CZT was proposed in [19] and this transform can produce a spectrum with denser frequency samples at any required place. Furthermore, the warped discrete Fourier transform which can produce inhomogeneous frequency samples was studied in $[20,21]$. These algorithms may have some value in the LSS target detection, but the computational complexity is very high. More seriously, the above methods do not consider the characteristics of existing radar systems and the convenience of retrofitting process.

A new detection algorithm based on spectrum zoom processing is proposed in this paper according to the characteristics of LSS targets. By retrofitting the existing radar systems with some simple operations, the proposed algorithm can make the radar systems obtain an excellent LSS target detection ability. Meanwhile, the high-speed target detection ability is still retained. With the available rangeDoppler frequency image in the existing radar systems, the proposed algorithm firstly concatenates the data from the same low Doppler frequency slot of different images and then performs the spectrum zoom processing on the obtained data. Finally, the clutter suppression and target detection are performed on the spectrum zoom result. Spectrum zoom processing is the key step which can effectively separate the target echo and ground clutter in the frequency domain and significantly improve the signal-to-clutter ratio (SCR).

This paper is organized as follows. In Section 2, the basic theory of spectrum zoom processing is introduced. In Section 3, the spectrum zoom processing based LSS target detection algorithm is proposed. In Section 4, the performance of the proposed algorithm is theoretically analyzed in terms of the SCR improvement and computational load. In Section 5, we verify the theoretical analysis in Section 4 with some real data. Finally, conclusions are drawn in Section 6.

\section{Basic Theory of Spectrum Zoom Processing}

2.1. Signal Model. Let $s(t), 0 \leq t<M N T_{s}$ be a continuous time complex signal with a finite length, where $M$ and $N$ are positive integers and $T_{s}>0$. Dividing $s(t)$ into $M$ signals $s_{0}(t), s_{1}(t), \ldots, s_{M-1}(t)$ with the length of $N T_{s}$, we can get

$$
s(t)=s_{0}(t)+s_{1}(t)+\cdots+s_{M-1}(t),
$$

where

$$
s_{m}(t)= \begin{cases}s(t) & m N T_{s} \leq t<(m+1) N T_{s}, \\ 0 & \text { other, } \\ & m=0,1, \ldots, M-1 .\end{cases}
$$

Shifting the signal $s_{m}(t)$ to $0 \leq t<N T_{s}$ along the time axis, we have

$$
x_{m}(t)=s_{m}\left(t+m N T_{s}\right) .
$$

Combining (1) with (3), we can obtain

$$
\begin{aligned}
s(t)= & x_{0}(t)+x_{1}\left(t-N T_{s}\right)+\cdots \\
& +x_{M-1}\left(t-(M-1) N T_{s}\right) .
\end{aligned}
$$

After sampling the continuous time signal $s(t)$ and $x_{m}(t)$ with a period of $T_{s}$, respectively, the resulting discrete time sequences are

$$
\begin{gathered}
s[n]=s\left(n T_{s}\right), \quad n=0,1, \ldots, M N-1, \\
x_{m}[n]=x_{m}\left(n T_{s}\right), \quad n=0,1, \ldots, N-1 .
\end{gathered}
$$

Obviously, $s[n]$ is just the arrangement of $x_{0}[n], x_{1}[n], \ldots$, $x_{M-1}[n]$ with the sequential order.

According to [22], the Fourier transform (FT) of $s(t)$ can be written as

$$
S(\omega)=\int_{-\infty}^{\infty} s(t) e^{-\mathrm{j} \omega t} \mathrm{~d} t .
$$


Meanwhile, the discrete time Fourier transform (DTFT) of $s[n]$ can be written as

$$
S(\Omega)=\sum_{n=0}^{M N-1} s[n] e^{-\mathrm{j} \Omega n},
$$

and the discrete Fourier transform (DFT) of $s[n]$ can be written as

$$
S[k]=\sum_{n=0}^{M N-1} s[n] W_{M N}^{k n},
$$

where $W_{M N}=e^{-\mathrm{j} 2 \pi /(M N)}$ and $k=0,1, \ldots, M N-1$.

Obviously, the sequence $S[k]$ is just the sample of $S(\Omega)$ at the frequency $f=k \Delta f$; that is,

$$
S[k]=\left.S(\Omega)\right|_{f=k \Delta f},
$$

where $\Delta f=F_{s} /(M N)$. Assume that the whole energy of $s(t)$ is concentrated in $f \in\left[0, F_{s}\right]$. Therefore, the sampling process of $s(t)$ obeys Nyquist's law, and $S(\Omega)$ is the extension of $S(\omega)$ with the period of $f=F_{s}$. In this case, $S[k]$ is also the sample of $S(\omega)$ at $f=k \Delta f$; that is,

$$
S[k]=\left.S(\Omega)\right|_{f=k \Delta f}=\left.S(\omega)\right|_{f=k \Delta f} .
$$

Similar to (6)-(10), the FT of $x_{m}(t)$ can be written as

$$
X_{m}(\omega)=\int_{-\infty}^{\infty} x_{m}(t) e^{-\mathrm{j} \omega t} \mathrm{~d} t
$$

Meanwhile, the DTFT and DFT of $x_{m}[n]$ can, respectively, be written as

$$
\begin{aligned}
X_{m}(\Omega) & =\sum_{n=0}^{N-1} x_{m}[n] e^{-\mathrm{j} \Omega n}, \\
X_{m}[k] & =\sum_{n=0}^{N-1} x_{m}[n] W_{N}^{k n},
\end{aligned}
$$

where $m=0,1, \ldots, M-1, k=0,1, \ldots, N-1$ and $W_{N}=$ $e^{-\mathrm{j} 2 \pi / N}$. We also have

$$
X_{m}[k]=\left.X_{m}(\Omega)\right|_{f=k \Delta f}=\left.X_{m}(\omega)\right|_{f=k \Delta f},
$$

where $\Delta f=F_{s} / N$.

In addition, taking the FT on both sides of (4), we arrive at

$$
\begin{aligned}
S(\omega)= & X_{0}(\omega)+X_{1}(\omega) e^{-\mathrm{j} \omega N T_{s}}+\cdots \\
& +X_{M-1}(\omega) e^{-\mathrm{j} \omega(M-1) N T_{s}} \\
= & \sum_{m=0}^{M-1} X_{m}(\omega) e^{-\mathrm{j} \omega m N T_{s}},
\end{aligned}
$$

which shows the relationship between $s(t)$ and $x_{0}(t), x_{1}(t)$, $\ldots, x_{M-1}(t)$ in the frequency domain.
2.2. Spectrum Zoom Processing. The sequence $S[k], k=0,1$, $\ldots, M N-1$, shows the whole spectrum information of $s(t)$ in $\left[0, F_{s}\right]$. However, only partial spectrum information of $s(t)$ is necessary in some applications. Consider a specific problem. Assume that the sequence $X_{m}[k], k=0,1, \ldots, N-1$, is known. Then, divide the frequency range $\left[0, F_{s}\right]$ into $N$ bands with equal lengths. In this case, how to obtain the spectrum information of $s(t)$ at the $p$ th band, that is, $S[p M], S[p M+$ $1], \ldots, S[p M+M-1], p \in\{0,1, \ldots, N-1\}$ ?

An obvious method is as follows: firstly calculate the sequence $S[k], k=0,1, \ldots, M N-1$, according to (8), and then select out $S[p M], S[p M+1], \ldots, S[p M+M-1]$. Here, we introduce a fast method for obtaining $S[p M], S[p M+$ $1], \ldots, S[p M+M-1]$ with $X_{m}[k], k=0,1, \ldots, N-1$, as follows. This method will be applied to retrofit the existing radar systems in Section 3.

For simplicity, let

$$
\begin{aligned}
& f_{0} \triangleq \frac{(p M+q) F_{s}}{M N}, \\
& \omega_{0} \triangleq 2 \pi f_{0}=\frac{2 \pi(p M+q) F_{s}}{M N},
\end{aligned}
$$

where $p=0,1, \ldots, N-1$ and $q=0,1, \ldots, M-1$. According to $(10)$, we have

$$
S[p M+q]=\left.S(\omega)\right|_{f=f_{0}}=\left.S(\omega)\right|_{\omega=\omega_{0}} .
$$

Substituting (15) into (17), we can get

$$
\begin{aligned}
S[p M+q] & =\sum_{m=0}^{M-1} X_{m}\left(\omega_{0}\right) e^{-\mathrm{j} \omega_{0} m N T_{s}} \\
& =\sum_{m=0}^{M-1} X_{m}\left(\omega_{0}\right) e^{-\mathrm{j}\left(2 \pi(p M+q) F_{s} / M N\right) m N T_{s}} \\
& =\sum_{m=0}^{M-1} X_{m}\left(\omega_{0}\right) e^{-\mathrm{j} 2 \pi p m} e^{-\mathrm{j} 2 \pi q m / M} \\
& =\sum_{m=0}^{M-1} X_{m}\left(\omega_{0}\right) W_{M}^{q m},
\end{aligned}
$$

where $W_{M}=e^{-\mathrm{j} 2 \pi / M}$. It can be seen that $S[p M+q]$ is just the DFT of $X_{0}\left(\omega_{0}\right), X_{1}\left(\omega_{0}\right), \ldots, X_{M-1}\left(\omega_{0}\right)$. Therefore, it is very easy to obtain $S[p M+q]$ by applying the sequence $X_{0}\left(\omega_{0}\right), X_{1}\left(\omega_{0}\right), \ldots, X_{M-1}\left(\omega_{0}\right)$.

Since $\omega_{0}$ includes the variable $q$, we need to calculate a new sequence $X_{0}\left(\omega_{0}\right), X_{1}\left(\omega_{0}\right), \ldots, X_{M-1}\left(\omega_{0}\right)$ for a given $q$, which can result in a high computational load. Instead, we can approximate $\omega_{0}$ as follows:

$$
\omega_{0} \approx \frac{2 \pi p M F_{s}}{M N}=\frac{2 \pi p F_{s}}{N} \triangleq \widehat{\omega}_{0} .
$$

Equation (18) can be approximated as

$$
S[p M+q] \approx \sum_{m=0}^{M-1} X_{m}\left(\widehat{\omega}_{0}\right) W_{M}^{q m} .
$$


According to (14), we can get

$$
X_{m}[p]=\left.X_{m}(\omega)\right|_{\omega=2 \pi p F_{s} / N}=X_{m}\left(\widehat{\omega}_{0}\right) .
$$

Substituting (21) into (20), we can get

$$
S[p M+q] \approx \sum_{m=0}^{M-1} X_{m}[p] W_{M}^{q m} .
$$

Hence, $S[p M+q]$ can be approximated as the DFT of $X_{0}[p], X_{1}[p], \ldots, X_{M-1}[p]$. Since the variable $q$ is not contained in the sequence $X_{0}[p], X_{1}[p], \ldots, X_{M-1}[p]$, for a given $p$, only the same sequence $X_{0}[p], X_{1}[p], \ldots, X_{M-1}[p]$ needs to be calculated when $q$ takes all the $0,1, \ldots, M-1$. This way can significantly reduce the computational load. In the case when $X_{m}[k], k=0,1, \ldots, N-1$, is known, a fast way for obtaining the spectrum information of $s(t)$ at the $p$ th band (i.e., $S[p M], S[p M+1], \ldots, S[p M+M-1])$ is to select out the sequence $X_{0}[p], X_{1}[p], \ldots, X_{M-1}[p]$ from the known information and then perform the DFT.

According to (10) and (14), $X_{m}[k], k=0,1, \ldots, N-1$ is the sample of $X_{m}(\Omega)$ with the period of $F_{s} / N$, whereas $S[k], k=0,1, \ldots, M N-1$, is the sample of $S(\Omega)$ with the period of $F_{s} /(M N)$. From (22), we know that the frequency spectrum sample $S[k]$ with a short sampling period can be obtained from the frequency spectrum sample $X_{m}[k]$ with a relative longer sampling period by DFT, which is the socalled spectrum zoom processing. According to the above analysis, we can summarize the spectrum zoom processing as follows: (i) sample the continuous time complex signal $s(t)$ whose frequency spectrum locates in $\left[0, F_{s}\right]$ with a period of $T_{s}$, and obtain $M$ sequences $x_{m}[n], n=0,1, \ldots, N-$ 1 with the length $N$; (ii) write the DFT of $x_{m}[n], n=$ $0,1, \ldots, N-1$ as $X_{m}[k], k=0,1, \ldots, N-1$; (iii) for an arbitrary $p \in\{0,1, \ldots, N-1\}$, the DFT of sequence $X_{0}[p], X_{1}[p], \ldots, X_{M-1}[p]$ can be approximately regarded as the spectrum sampling result of $s(t)$ in $\left[p F_{s} / N,(p+1) F_{s} / N\right)$ with a period of $F_{s} /(M N)$ or can be equivalently regarded as the DFT of the sequence $s[n], n=0,1, \ldots, M N-1$, where $s[n]$ is the sequential arrangement of the $M$ sequences $x_{0}[n], x_{1}[n], \ldots, x_{M-1}[n], n=0,1, \ldots, N-1$, in the frequency range $\left[p F_{s} / N,(p+1) F_{s} / N\right)$ (i.e., $S[p M], S[p M+$ $1], \ldots, S[p M+M-1])$. It can be seen that the spectrum zoom processing provides a simple and fast approach for obtaining the refined spectrum from the coarse spectrum, which is very significant in the applications where only the refined spectrum on a partial band is necessary.

\section{Spectrum Zoom Processing Used for the LSS Target Detection}

The existing radar systems are mainly designed for highspeed targets. Considering the facts that the instantaneous position of the high-speed target varies quickly and the Doppler frequency of target echo varies in a board range, the detector usually takes a small coherent accumulation pulse number. Such an operation can obtain a high frame rate to update the target state quickly with a low computational complexity. Meanwhile, the resulting range-Doppler frequency image has a large spectrum interval. However, as a large number of LSS targets appear in recent years, the existing radar systems cannot perform an effective detection on these targets. The main reasons are as follows: (1) the echo of LSS target is very weak, and more coherent pulses are necessary to accumulate the target energy; (2) the Doppler frequency of LSS target echo is very close to that of the ground clutter, and the spectrum should be refined enough to separate them. Therefore, the existing radar systems should be retrofitted properly.

Based on the spectrum zoom processing introduced in Section 2, a simple and feasible scheme is designed for retrofitting the existing radar systems in this section. This scheme applies the available range-Doppler frequency image in the existing radar systems to obtain the refined spectrum information of the observation data in the low Doppler frequency band, which can effectively separate the target echo and ground clutter in the frequency domain and improve the accumulation effect of the target energy. Next, this retrofitting scheme will be introduced in detail.

3.1. Whole Retrofitting Scheme. A typical model of the pulse Doppler radar observation data is shown in Figure 1. The horizontal axis represents the fast time dimension, containing $L$ range gates in total. The vertical axis represents the slow time dimension, containing $M N$ pulses in total. Each sequential $N$ pulses are regarded as a frame of the observation data, and it contains $M$ frames of the observation data in Figure 1. Write the $n$th pulse as $\mathbf{z}_{n}=\left(z_{n, 1}, z_{n, 2}, \ldots, z_{n, L}\right)$, where $z_{n, l}$ represents the sample of the $l$ th range gate in the $n$th pulse, $n=1,2, \ldots, M N, l=1,2, \ldots, L$. Define $N \times L$ matrix

$$
\mathbf{Z}_{m}=\left(\begin{array}{c}
\mathbf{z}_{(m-1) N+1} \\
\mathbf{z}_{(m-1) N+2} \\
\vdots \\
\mathbf{z}_{m N}
\end{array}\right),
$$

which represents the $m$ th frame of the observation data, $m=$ $1,2, \ldots, M$.

Figure 2 shows the whole scheme for retrofitting the existing radar systems by the spectrum zoom processing. Only the operations after the pulse compression are considered. The top dashed box contains the original detection process of the existing radar systems which can effectively detect the highspeed target. The lower dashed box contains the concrete retrofitting operations on the existing radar systems. It can be seen that all the original processing operations are retained and only some simple operations including data selection, DFT, and detection are added in this retrofitting scheme.

3.2. Original Detection Process. The detection process in the existing radar systems are mainly used to detect the high-speed target. Firstly, perform the coherent accumulation. Specifically, perform the DFT on each frame of the observation data along the slow time axis. In fact, this is usually realized by the fast Fourier transform (FFT). After the coherent accumulation, we can obtain $M$ frames of $N \times L$ 


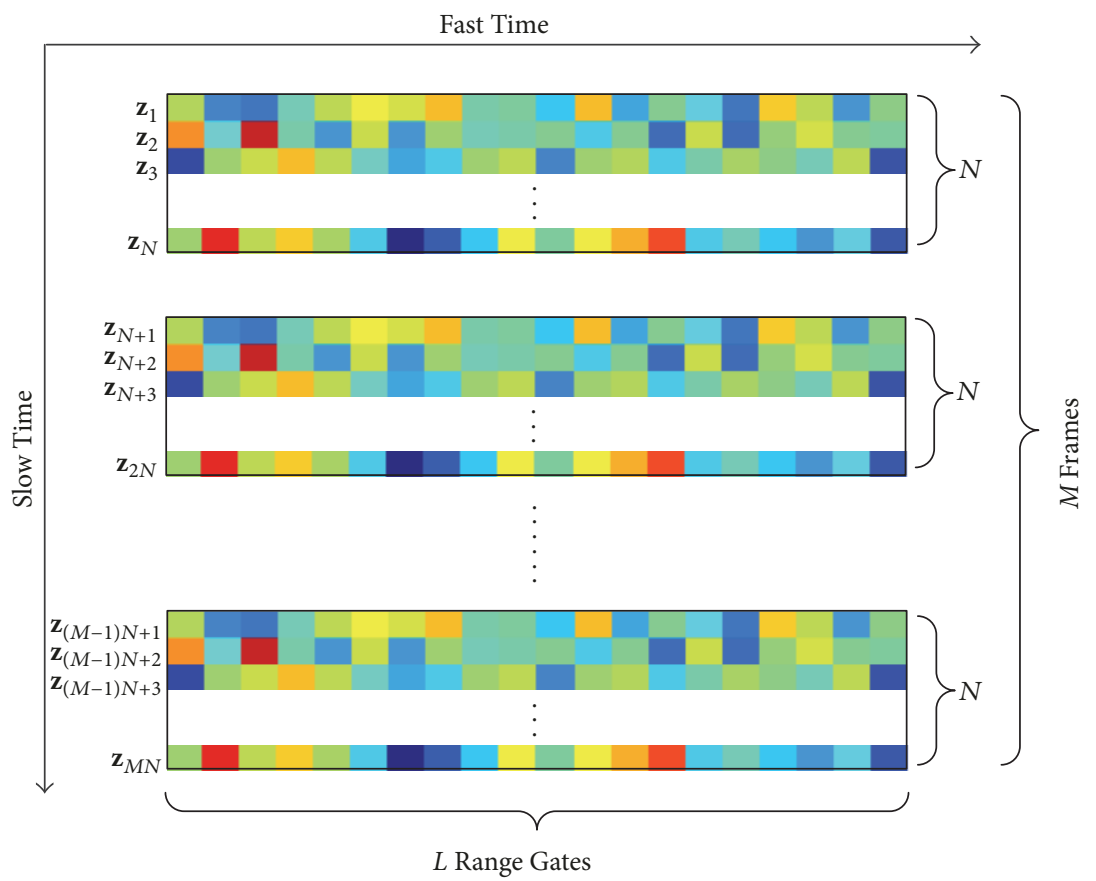

FIgURE 1: Typical model of the pulse Doppler radar observation data.

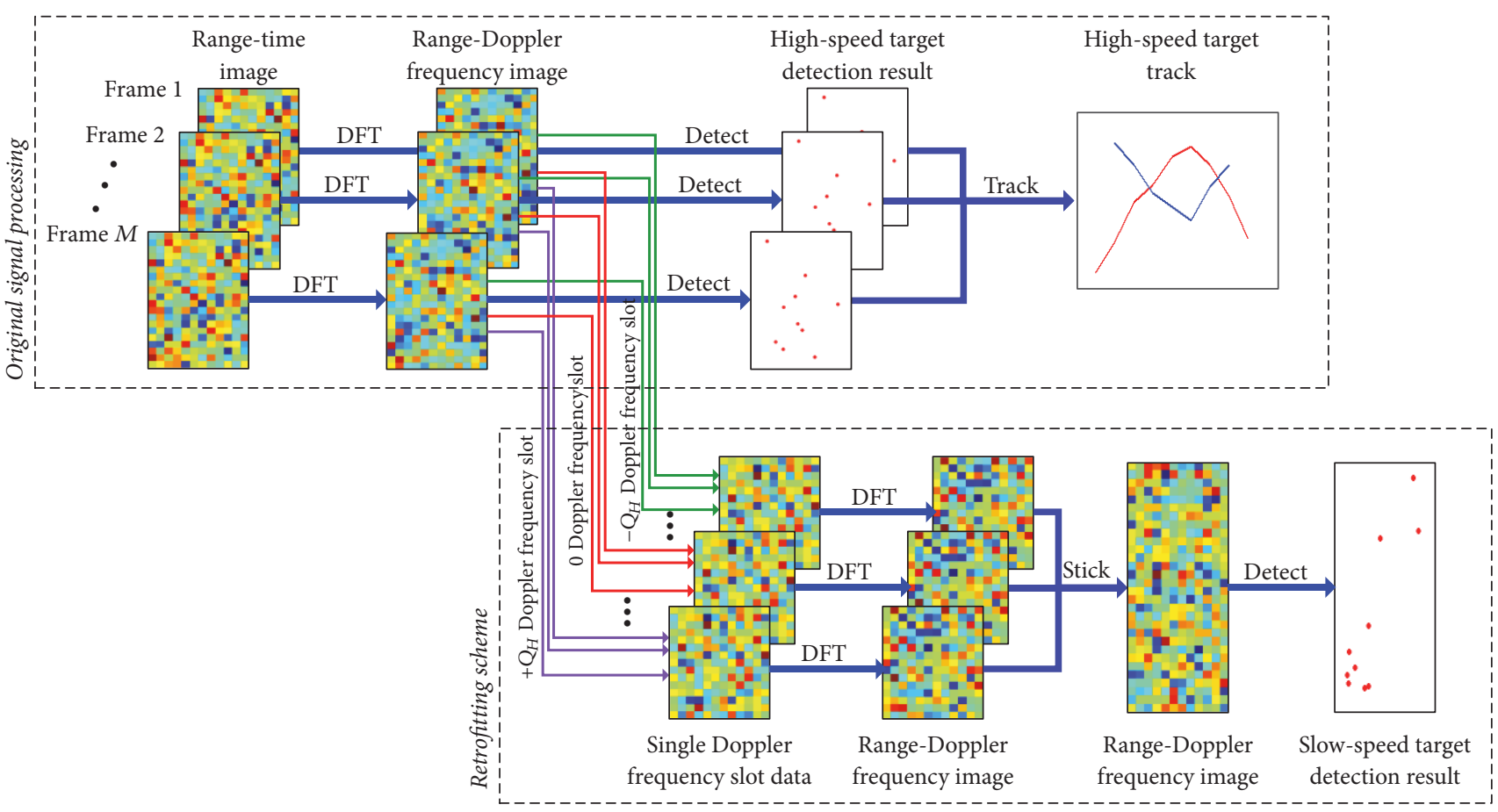

FIGURE 2: Whole scheme for retrofitting the existing radar systems by the spectrum zoom processing.

range-Doppler frequency images. The $m$ th frame of rangeDoppler frequency image can be written as

$$
\mathbf{X}_{m}=\left(\mathbf{x}_{m, 1}, \mathbf{x}_{m, 2}, \ldots, \mathbf{x}_{m, L}\right)
$$

where

$$
\mathbf{x}_{m, l}=\left(x_{(m-1) N+1, l}, x_{(m-1) N+2, l}, \ldots, x_{m N, l}\right)^{\mathrm{T}} .
$$

Then, perform the target detection on each frame of range-Doppler frequency image. The Doppler frequency of the high-speed target echo is much higher than that of the ground clutter. Hence, it is very easy to separate them in the frequency domain. In general, a simple CFAR algorithm can find out the potential high-speed target. Finally, when the detection results from the multiple frames of range-Doppler 
frequency images are obtained, we can jointly analyze these results to improve the detection performance and estimate the target track.

3.3. Concrete Retrofitting Operations. To obtain the LSS target detection ability for the radar systems, some proper retrofitting operations are necessary. Here, the spectrum zoom processing is mainly used to realize the two points: (1) separate the LSS target echo and the ground clutter in the frequency domain; (2) improve the SCR.

Before introducing the concrete retrofitting operations, some necessary analysis will be made as follows. The velocity of most LSS targets is no more than $v_{\max }=200 \mathrm{~km} / \mathrm{h}$. This prior information can be applied to reduce the target detection range. According to [23], the Doppler frequency of target echo can be expressed as

$$
f_{d}=\frac{2 v}{\lambda}
$$

where $v$ denotes the radial velocity, and $\lambda$ denotes the wavelength of radar transmission signal. Write the pulse repetition frequency of the observation data in Figure 1 as $F_{s}$. Then, the interval of two adjacent Doppler frequency slots in the range-Doppler frequency image of existing radar systems is $\Delta f=F_{s} / N$. According to (26), the difference of radial velocities corresponding to two adjacent Doppler frequency slots is $\Delta v=\lambda \Delta f / 2$, and the radial velocity corresponding to each Doppler frequency slot can, respectively, be written as $-(N / 2) \Delta v,-(N / 2-1) \Delta v, \ldots,(N / 2-1) \Delta v$. Hence, the Doppler frequency slot index corresponding to the maximal velocity $v_{\max }$ of LSS targets is

$$
Q_{H}=\operatorname{round}\left(\frac{v_{\max }}{\Delta v}\right),
$$

where the function $\operatorname{round}(x)$ represents the rounding of $x$. In the existing radar systems, since $\Delta v$ is often large, we know that $Q_{H}$ is small. Generally, $Q_{H} \leq 5$. The Doppler frequencies and radial velocities in the $k=-Q_{H},-Q_{H}+$ $1, \ldots, Q_{H}$ Doppler frequency slots are very low, so these Doppler frequency slots are called the low Doppler frequency (LDF) area. Meanwhile, the rest of Doppler frequency slots are called the high Doppler frequency (HDF) area.

To obtain the enough elevating force, most LSS targets have a minimal velocity denoted as $v_{\min }$. In general, $v_{\min }$ is comparable to $\Delta v$. Therefore, we can believe that the distribution range of LSS target echo is the whole LDF area. The ground clutter consists of the echoes of ground, buildings, and trees. Generally, the ground clutter mainly locates near the $k=0$ Doppler frequency slot. As a result, the echo of LSS target is very close to the ground clutter in the LDF area. In some cases, both of them may locate in the same Doppler frequency slot. This makes it very difficult to detect the LSS target and separating the echo of LSS target and the ground clutter is the key of this procedure. Next, the concrete retrofitting operations will be introduced.

Firstly, take out the data in the LDF area. Specifically, take out the data in the $k=Q_{H}$ Doppler frequency slot from the available $M$ frames of range-Doppler frequency images of the existing radar system, and concatenate the data together to form a $M \times L$ matrix. Meanwhile, perform the same operation on the data in the $k=-Q_{H},-Q_{H}+1, \ldots, Q_{H}-1$ Doppler frequency slots, respectively. In this way, we can obtain $2 Q_{H^{+}}$ 1 matrices with a dimension of $M \times L$.

Then, respectively, perform the DFT on the $2 Q_{H}+1$ matrices along the vertical axis such that $2 Q_{H}+1$ new $M \times L$ matrices are obtained. These new matrices are still rangeDoppler frequency images where the horizontal axis represents the range gate and the vertical axis represents the refined Doppler frequency slot. The range of Doppler frequency of the $k$ th image is $[(-1 / 2+k) \Delta f,(1 / 2+k) \Delta f], k=-Q_{H},-Q_{H}+$ $1, \ldots, Q_{H}$. Concatenate the $2 Q_{H}+1$ frames of range-Doppler frequency images to form a frame of $\left(2 Q_{H}+1\right) M \times L$ rangeDoppler frequency image. The range of Doppler frequency is $\left[-\left(1 / 2+Q_{H}\right) \Delta f,\left(1 / 2+Q_{H}\right) \Delta f\right]$, the interval of two adjacent Doppler frequency slots is $\Delta f / M$, and the corresponding velocity interval is $\Delta v / M$ in the $\left(2 Q_{H}+1\right) M \times L$ rangeDoppler frequency image. Compared with the range-Doppler frequency image in the existing radar system, the spectrum zoom degree improves $M$ times in the new range-Doppler frequency image obtained by the second DFT. In fact, this means that the separation degree of the ground clutter and the echo of LSS target improve $M$ times. In the new $\left(2 Q_{H}+\right.$ 1) $M \times L$ range-Doppler frequency image, the ground clutter is still near the zero Doppler frequency, whereas the echo of LSS target is away from the zero Doppler frequency. Hence, the echo of LSS target and the ground clutter are obviously separated in the frequency domain. In addition, the DFT is essentially a coherent accumulation process. An additional benefit of the second DFT is improving the SCR about $M$ times, enhancing the ability of detecting the LSS target.

In the $\left(2 Q_{H}+1\right) M \times L$ range-Doppler frequency image, the Doppler frequency slot index corresponding to the minimal velocity $v_{\min }$ of LSS targets is

$$
Q_{L}=\operatorname{round}\left(\frac{v_{\min }}{\Delta v / M}\right)=\operatorname{round}\left(\frac{M v_{\min }}{\Delta v}\right) .
$$

Hence, there is no echo of the LSS target in the Doppler frequency slots whose indexes are $-\left(Q_{L}-1\right),-\left(Q_{L}-2\right), \ldots, Q_{L}-1$, whereas the ground clutter mainly locates in these Doppler frequency slots. To suppress the ground clutter, it is straightforward to eliminate these Doppler frequency slots from the $\left(2 Q_{H}+1\right) M \times L$ range-Doppler frequency image. Finally, perform the target detection on the rest of Doppler frequency slots with the common detection algorithms and a LSS target detection result is obtained. In the spectrum zoom based retrofitting scheme, we can obtain a LSS target detection result whenever $M$ frames of observation data are received. Such a detection output rate can meet the requirement of the LSS target detection, because the position of LSS target varies very slowly. When multiple sets of detection results are obtained, we can jointly analyze these results to estimate the track of LSS target.

The spectrum zoom based retrofitting scheme retains all the original processing operations of the existing radar 
systems and makes full use of the available range-Doppler frequency images. The concrete retrofitting operations only includes data selection, DFT, and threshold detection which are very easy to implement with hardware. Overall, the spectrum zoom based target detection algorithm can make the radar system obtain a good ability of LSS target detection, while the original ability of high-speed target detection can still be retained.

\section{Performance Analysis}

This section analyzes the performance of the spectrum zoom based LSS target detection algorithm from the SCR improvement and computational load. Two traditional detection algorithms are selected as the reference algorithms. The first reference algorithm is implemented as follows: take $M$ successive frames of the observation data (i.e., $M N$ pulses) as a whole; and then perform the DFT along the time dimension to obtain a $M N \times L$ range-Doppler frequency image; finally perform the target detection. This algorithm only performs the DFT once. Hence, it can be called the one DFT based algorithm. The other reference algorithm is realized as follows: take $M$ successive frames of the observation data as a whole; and then perform the CZT along the time dimension to obtain a refined range-Doppler frequency image where the corresponding velocity range is $\left[-v_{\max }, v_{\max }\right]$; finally perform the target detection. This algorithm is called the CZT based algorithm.

The original intention of this paper is to retrofit the existing radar system to obtain the ability of LSS target detection. Hence, the performance analysis is performed in this specific application scene. Firstly, consider the performance of the one DFT based algorithm, the CZT based algorithm, and the spectrum zoom based algorithm in the SCR improvement. The one DFT based algorithm considers the $M$ frames of the observation data as a whole and performs the coherent accumulation. In theory, this algorithm can improve the SCR with $M N$ times. The range-Doppler frequency image obtained by the CZT based algorithm is just the area corresponding to the velocity range $\left[-v_{\max }, v_{\max }\right]$ of the range-Doppler frequency image obtained by the one DFT based algorithm. Hence, the CZT based algorithm can also improve the SCR with $M N$ times. The spectrum zoom based algorithm contains a two-stage coherent accumulation process. The first stage which is an original operation in the existing radar system can improve the SCR with $N$ times. The second stage is introduced in retrofitting the radar system with the spectrum zoom processing. The SCR is improved less than $M$ times slightly in the second stage because of the approximation in (22). Therefore, the SCR improvement capacity of the spectrum zoom based algorithm is less than $M N$ times slightly.

Next, consider the performance of the three algorithms in computational load. In order to simplify the analysis, assume that $M$ is 2 to the $\kappa_{1}$ th power and $N$ is 2 to the $\kappa_{2}$ th power, where $\kappa_{1}$ and $\kappa_{2}$ are integers. In addition, assume all the DFT is implemented by FFT. Take the $M$ frames of the observation data as an example for performing the target detection.
In the coherent accumulation process, according to [22], the number of complex additions and the number of complex multiplications required in the one DFT based algorithm are

$$
\begin{aligned}
& b_{1}=L \times(M N) \log _{2}(M N), \\
& b_{2}=L \times\left(\frac{M N}{2}\right) \log _{2}(M N) .
\end{aligned}
$$

In the CZT based algorithm, the sampling number of the time-domain signals is $M N$ in each range gate, and the sampling number of the Doppler frequencies is

$$
H=\operatorname{round}\left(\frac{2 v_{\max }}{\Delta v / M}\right) .
$$

The length of DFT is $\Gamma=2 M N$ in the CZT process, because $M$ and $N$ are 2 to the integer powers. According to [18, 19], the number of complex additions and the number of complex multiplications required in the CZT based algorithm are

$$
\begin{aligned}
& c_{1}=2 L \Gamma \log _{2} \Gamma, \\
& c_{2}=L\left(\Gamma \log _{2} \Gamma+H+M N+\Gamma\right) .
\end{aligned}
$$

The spectrum zoom based algorithm is performed on the basis of the existing $N \times L$ range-Doppler frequency images. According to (27), the spectrum zoom processing only needs to be implemented on the $k=-Q_{H},-Q_{H}+1, \ldots, Q_{H}$ Doppler frequency slots. Therefore, the number of complex additions and the number of complex multiplications required in the spectrum zoom based algorithm are

$$
\begin{aligned}
& d_{1}=L\left(2 Q_{H}+1\right) \times M \log _{2} M, \\
& d_{2}=L\left(2 Q_{H}+1\right) \times\left(\frac{M}{2}\right) \log _{2} M .
\end{aligned}
$$

Assume that the computational load required by a complex multiplication and a complex addition is equal. Also, we assume that $M$ and $N$ are much larger than 1 . For simplicity, take $M=N$. In this case, the computational load of the one DFT based algorithm in the coherent accumulation process is

$$
b=b_{1}+b_{2}=3 L M^{2} \log _{2} M .
$$

The computational load of the CZT based algorithm is

$$
\begin{aligned}
c & =c_{1}+c_{2}=L\left(3 \Gamma \log _{2} \Gamma+H+M N+\Gamma\right) \\
& =9 L M^{2}+12 L M^{2} \log _{2} M \approx 12 L M^{2} \log _{2} M .
\end{aligned}
$$

The computational load of the spectrum zoom based algorithm is

$$
\begin{aligned}
d & =d_{1}+d_{2}=1.5\left(2 Q_{H}+1\right) L M \log _{2} M \\
& \approx 3 Q_{H} L M \log _{2} M .
\end{aligned}
$$

It can be seen from comparing (33) with (35) that the computational load of the spectrum zoom based algorithm is only $Q_{H} / M$ of that of the one DFT based algorithm. 
According to the analysis in Section $3, Q_{H}$ is usually very small. Hence, the computational load of the spectrum zoom based algorithm is much lower than that of the one DFT based algorithm. By comparing (34) with (35), we can find that the computational load of the spectrum zoom based algorithm is also much lower than that of the CZT based algorithm. The main reason is because the spectrum zoom based algorithm can make full use of the available rangeDoppler frequency images in the existing radar system. Therefore, the proposed spectrum zoom based algorithm is more suitable for retrofitting the existing radar system.

The range-Doppler frequency images obtained by the above three algorithms have the same spectrum zoom degree, and every algorithm only needs to perform the target detection on the area corresponding to the velocity range $\left[-v_{\max }, v_{\max }\right]$. Therefore, the total numbers of the detected cells for the three algorithms are equal. This means that the three algorithms require the same computational load in the target detection process.

In summary, the SCR improvement capacity of the spectrum zoom based algorithm is similar to that of the one DFT based algorithm and the CZT based algorithm. However, the spectrum zoom based algorithm requires a much lower computational load, because this algorithm can make full use of the available coarse range-Doppler frequency images.

\section{Simulation Results with Real Data}

To test and verify the effectiveness of the spectrum zoom based detection algorithm, we choose a real data from the ground-based radar. The real data contains 3200 frames of the observation data in total, and each frame of the observation data contains $N=64$ pulses. The pulse repetition period is $T_{p}=18 \mu \mathrm{s}$ and the sampling rate is $f_{s}=160 \mathrm{MHz}$. There are $N_{r}=300$ range gates in total, and the length of a range gate is $\Delta=0.9375 \mathrm{~m}$. There are two small targets flying at a low altitude in the observation area. One is a slow target marked as Target 1 , and the other is a fast target marked as Target 2 . Target 1 moves away from the radar with a constant velocity $v_{1}=4.35 \mathrm{~m} / \mathrm{s}$. It started in the $173 \mathrm{rd}$ range gate at the initial time, and stopped in the 190th range gate at the terminal time. Meanwhile, Target 2 moves away from the radar with a constant velocity $v_{2}=50.13 \mathrm{~m} / \mathrm{s}$. It started in the 47 th range gate at the initial time and stopped in the 244th range gate at the terminal time.

In order to detect the high-speed target, the existing radar systems separately process each frame of the observation data. Figure 3 shows the coherent accumulation result of the first frame of the observation data in the existing radar system. It can be seen that both the echo of Target 1 and the ground clutter locate near the $k=0$ Doppler frequency slot. We cannot distinguish the echo of Target 1 and the ground clutter according to their spectrum characteristics in Figure 3. The echo of Target 2 locates in the $k=-5$ Doppler frequency slot. Meanwhile, the HDF area is free of the ground clutter, which is consistent with the analysis in Section 3.

Figure 4 shows the refined range-Doppler frequency images obtained from the first $M=32$ frames of the observation data for the one DFT based algorithm, the
TABLE 1: Running time for different algorithms.

\begin{tabular}{lc}
\hline & Running time (s) \\
\hline One DFT based algorithm & 36.23 \\
CZT based algorithm & 80.67 \\
Spectrum zoom based algorithm & 6.12 \\
\hline
\end{tabular}

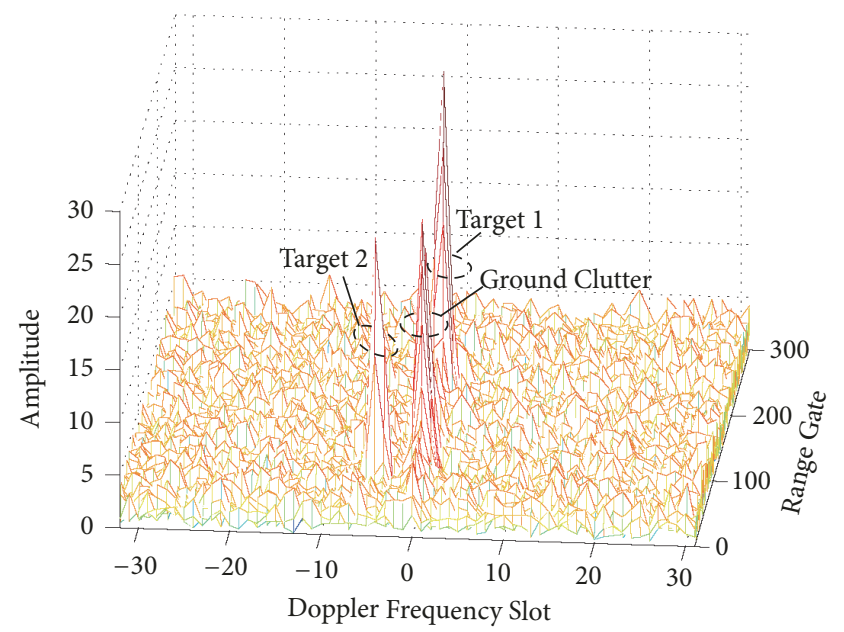

FIGURE 3: Range-Doppler frequency image obtained by the existing radar system.

CZT based algorithm, and the proposed spectrum zoom based algorithm. Figure 4(a) is the result of the one DFT based algorithm, Figure 4(b) is the result of the CZT based algorithm, and Figure 4(c) is the result of the spectrum zoom based algorithm. In order to show the echo of Target 1 and the ground clutter clearly, only the 64 Doppler frequency slots near the zero Doppler frequency are plotted in each image. It can be observed that the range-Doppler frequency images obtained by the three algorithms are very similar. The spectrum zoom degree is very high, and the echo of Target 1 and the ground clutter are well separated in the three range-Doppler frequency images. The heavy ground clutter mainly locates near the $k=0$ Doppler frequency slot, whereas the echo of Target 1 locates in the $k=-13$ Doppler frequency slot and the 173rd range gate. Comparing Figures 4(a) and 4(b) with Figure 4(c), we can find that the spectrum zoom based algorithm has similar performance with the classic algorithms in separating the echo of LSS target and the ground clutter. The amplitudes of Target $1^{\prime}$ echo are, respectively, 209, 209, and 203 in Figures 4(a), 4(b), and 4(c). This implies that the performance of the spectrum zoom based algorithm is very close to the classic algorithms in the energy accumulation.

The running time of processing the first $M=32$ frames of the observation data by the one DFT based algorithm, the CZT based algorithm, and the spectrum zoom based algorithm is shown in Table 1. The simulation is performed on a personal computer and programmed with MATLAB. It can be seen that the running time of the spectrum zoom based algorithm is only about $1 / 6$ of that of the one DFT based 


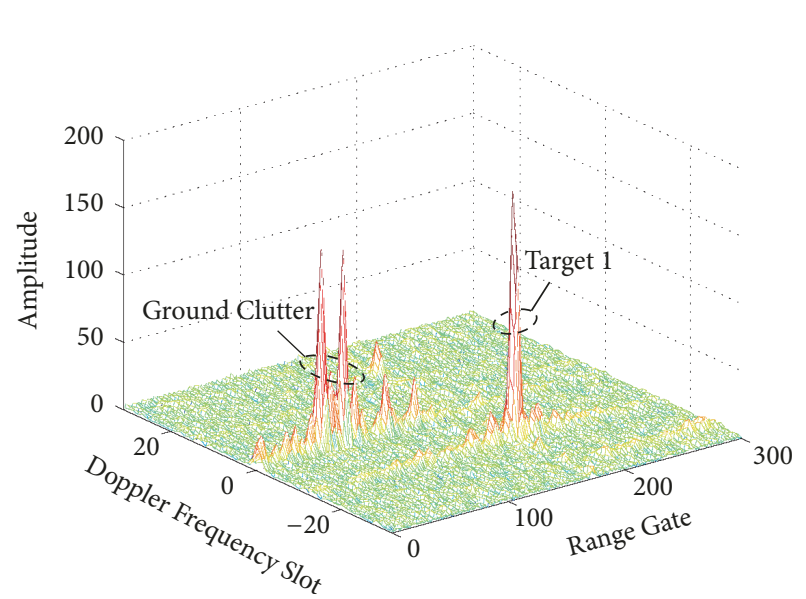

(a)

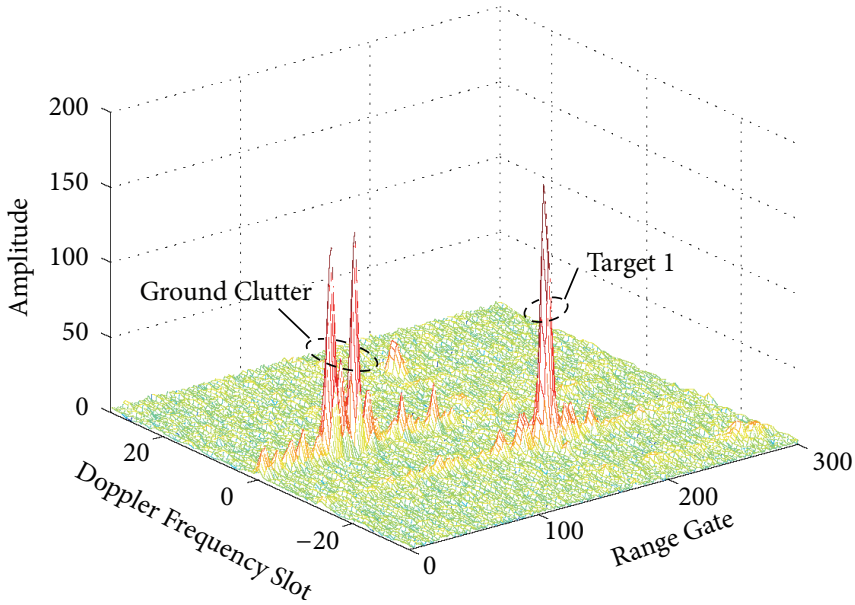

(b)

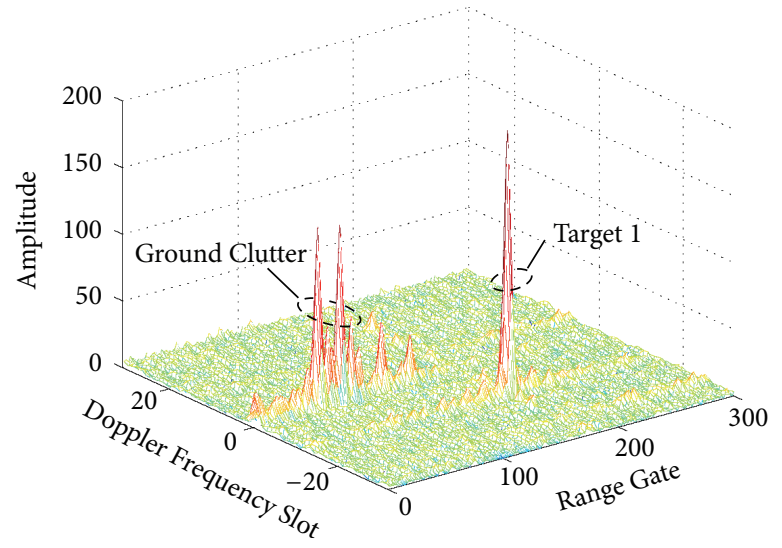

(c)

FIGURE 4: Refined range-Doppler frequency images for different algorithms: (a) one DFT based algorithm; (b) CZT based algorithm; (c) spectrum zoom based algorithm.

algorithm and $1 / 13$ of that of the CZT based algorithm. This illustrates that the spectrum zoom based algorithm requires a much lower computational load than the one DFT based algorithm and the CZT based algorithm.

According to the results shown in Figures 3 and 4 and Table 1, we can conclude that the proposed spectrum zoom based detection algorithm has similar performance with the one DFT based algorithm and the CZT based algorithm in refining spectrum, improving $\mathrm{SCR}$, and detecting targets, but the computational load is much lower than the existing two methods.

\section{Conclusions}

The existing radar systems which are mainly designed for the high-speed targets cannot perform an effective detection on the LSS targets whose number is growing fast in recent years. For this problem, a spectrum zoom processing based target detection algorithm is proposed in this paper to retrofit the existing radar systems. This algorithm can make full use of the available range-Doppler frequency images obtained in the existing radar systems, and the implementation of this algorithm is easy to follow. Firstly concatenate the data from the same Doppler frequency slot of different images, and then perform the spectrum zoom processing. Finally, the clutter suppression and target detection are performed. The proposed algorithm can obtain the same spectrum zoom degree with the one DFT based algorithm and the CZT based algorithm, but it only requires a much lower computational load. More importantly, only a few retrofitting operations are needed to retrofit the existing radar systems with the proposed algorithm. Therefore, the spectrum zoom processing based target detection algorithm is more suitable for retrofitting the existing radar systems.

\section{Conflicts of Interest}

The authors declare that they have no conflicts of interest.

\section{Acknowledgments}

This work was supported by the National Natural Science Foundation of China (Grant no. 61471019). 


\section{References}

[1] M. Skolnik, G. Linde, and K. Meads, "Senrad: An advanced wideband air-surveillance radar," IEEE Transactions on Aerospace and Electronic Systems, vol. 37, no. 4, pp. 1163-1175, 2001.

[2] J. M. Loomis, "Army Radar Requirements for the 21st Century," in Proceedings of the 2007 IEEE Radar Conference, pp. 1-6, Waltham, MA, USA, April 2007.

[3] X. Zhang, J. Sun, Y. Zhang, S. Lu, and C. Liu, "H-PMHT trackbefore-detect processing with DP-based track initiation and termination," IET Signal Processing, vol. 10, no. 9, pp. 1118-1125, 2016.

[4] X. Zhang, J. Sun, J. Fu, and S. Lu, "Fast implementation method of permutation test with valid strategy," Journal of Signal Processing, vol. 31, no. 10, pp. 1233-1239, 2015 (Chinese).

[5] X. Chen, J. Guan, Z. Bao, and Y. He, "Detection and extraction of target with micromotion in spiky sea clutter via short-time fractional fourier transform," IEEE Transactions on Geoscience and Remote Sensing, vol. 52, no. 2, pp. 1002-1018, 2014.

[6] S. P. Sira, D. Cochran, A. Papandreou-Suppappola et al., "Adaptive waveform design for improved detection of low-RCS targets in heavy sea clutter," IEEE Journal of Selected Topics in Signal Processing, vol. 1, no. 1, pp. 56-66, 2007.

[7] Y. Zhang, S. Qian, and T. Thayaparan, "Detection of a manoeuvring air target in strong sea clutter via joint time-frequency representation," IET Signal Processing, vol. 2, no. 3, pp. 216-222, 2008.

[8] L. Zuo, M. Li, X. Zhang, Y. Wang, and Y. Wu, "An efficient method for detecting slow-moving weak targets in sea clutter based on time-frequency iteration decomposition," IEEE Transactions on Geoscience and Remote Sensing, vol. 51, no. 6, pp. 3659-3672, 2013.

[9] A. Yasotharan and T. Thayaparan, "Time-frequency method for detecting an accelerating target in sea clutter," IEEE Transactions on Aerospace and Electronic Systems, vol. 42, no. 4, pp. 1289-1310, 2006.

[10] P. Suresh, T. Thayaparan, and K. Venkataramaniah, "FourierBessel transform and time-frequency-based approach for detecting manoeuvring air target in sea-clutter," IET Radar, Sonar \& Navigation, vol. 9, no. 5, pp. 481-491, 2015.

[11] A. Aprile, E. Grossi, M. Lops, and L. Venturino, "Track-beforedetect for sea clutter rejection: Tests with real data," IEEE Transactions on Aerospace and Electronic Systems, vol. 52, no. 3, pp. 1035-1045, 2016.

[12] L. Hao and Z. Lefeng, "Low velocity small radar target detection in maritime environment," in Proceedings of the 2010 2nd International Conference on Signal Processing Systems (ICSPS), pp. V3-385-V3-388, Dalian, China, July 2010.

[13] J. D. Park and J. F. Doherty, "Track Detection of Low Observable Targets Using a Motion Model," IEEE Access, vol. 3, pp. 14081415, 2015.

[14] Z. Zhou, S. Zhigang, and W. Renbiao, "Method for detecting ground moving target with range migration," in Proceedings of the IET International Radar Conference 2009, pp. 141-141, Guilin, China.

[15] F.-F. Gu, Q. Zhang, Y.-C. Chen, W.-J. Huo, and J.-C. Ni, "Parametric Sparse Representation Method for Motion Parameter Estimation of Ground Moving Target," IEEE Sensors Journal, vol. 16, no. 21, pp. 7646-7652, 2016.

[16] J. Sun, Q. Li, X. Zhang, and W. Sun, "An Efficient Implementation of Track-Oriented Multiple Hypothesis Tracker Using
Graphical Model Approaches," Mathematical Problems in Engineering, vol. 2017, Article ID 8061561, 2017.

[17] X. Zhang, J. Sun, S. Lu, and G. Wang, "Non-parametric detector in nonhomogeneous clutter environments with knowledgeaided permutation test," IET Radar, Sonar \& Navigation, vol. 10, no. 7, pp. 1310-1318, 2016.

[18] L. R. Rabiner, R. W. Schafer, and C. M. Rader, "The chirp ztransform algorithm and its application," Bell System Technical Journal, vol. 48, no. 5, pp. 1249-1292, 1969.

[19] I. Sarkar and A. T. Fam, "The interlaced chirp Z transform," Signal Processing, vol. 86, no. 9, pp. 2221-2232, 2006.

[20] A. Makur and S. K. Mitra, "Warped discrete-Fourier transform: Theory and applications," IEEE Transactions on Circuits and Systems I: Fundamental Theory and Applications, vol. 48, no. 9, pp. 1086-1093, 2001.

[21] S. Franz, S. K. Mitra, J. C. Schmidt, and G. Doblinger, "Warped discrete Fourier transform: A new concept in digital signal processing," in EEE International Conference on Acoustics, Speech, and Signal Processing (ICASSP), vol. 2, pp. 1205-1208, Orlando, USA, May 2002.

[22] A. V. Oppenheim, R. W. Schafer, and J. R. Buck, Discrete-Time Signal Processing, Prentice-Hall, USA, 1999.

[23] M. A. Richards, Fundamentals of Radar Signal Processing, McGraw-Hill, USA, 2005. 


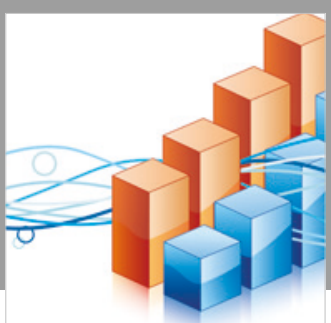

Advances in

Operations Research

\section{-n-m}
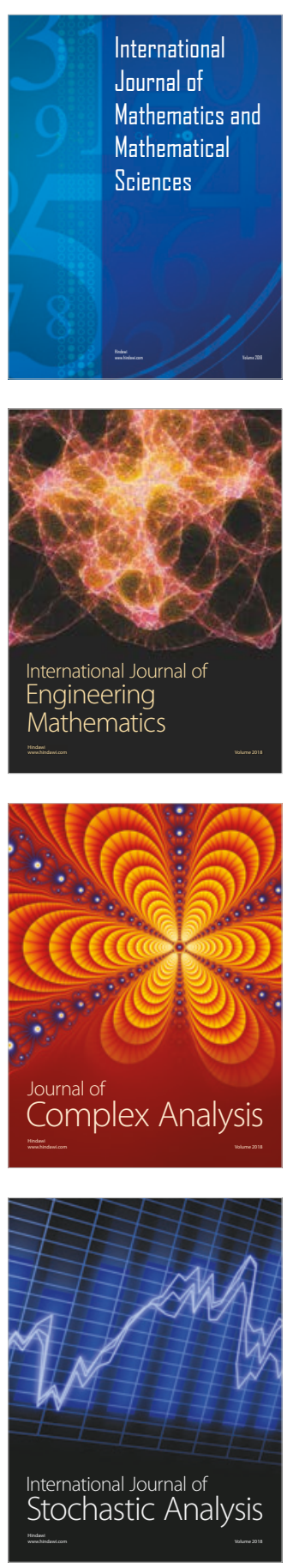
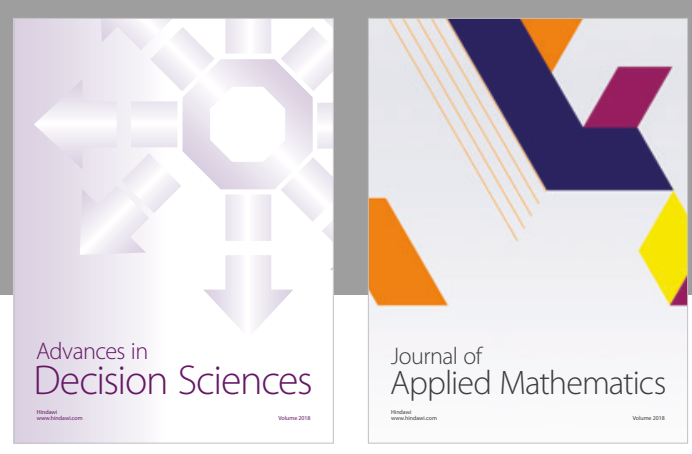

Journal of

Applied Mathematics
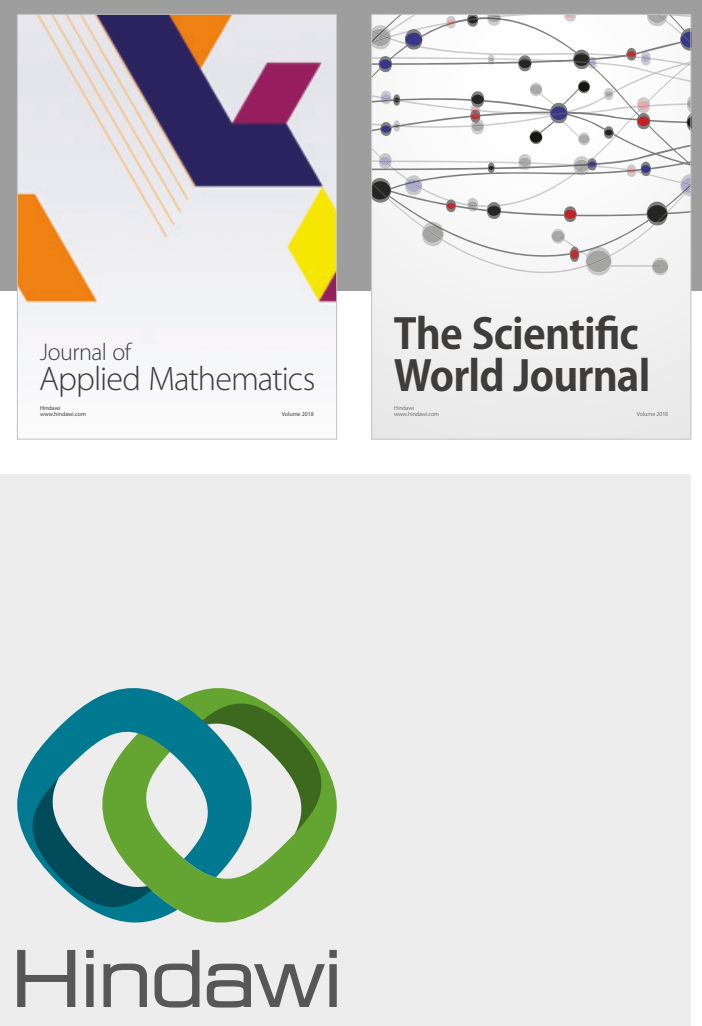

Submit your manuscripts at

www.hindawi.com

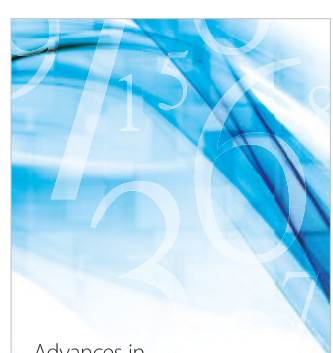

Advances in
Numerical Analysis
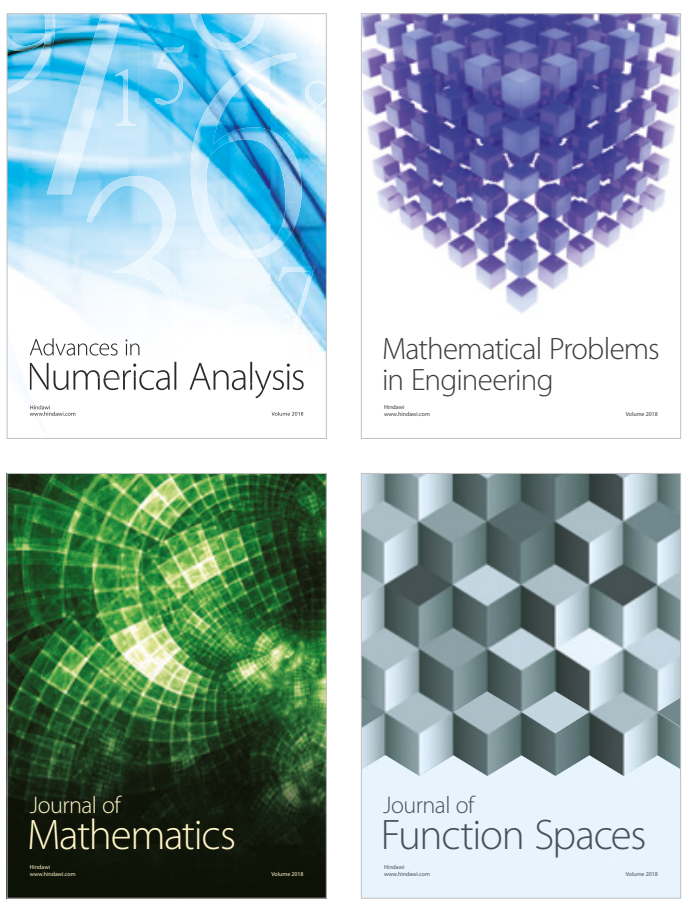

Mathematical Problems in Engineering

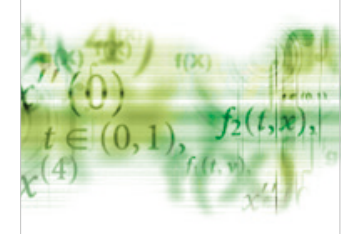

International Journal of

Differential Equations

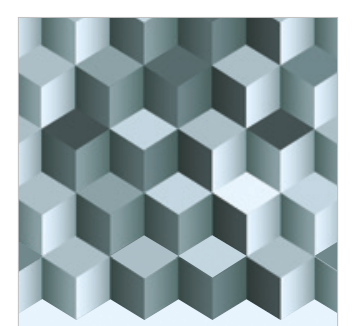

Journal of

Function Spaces

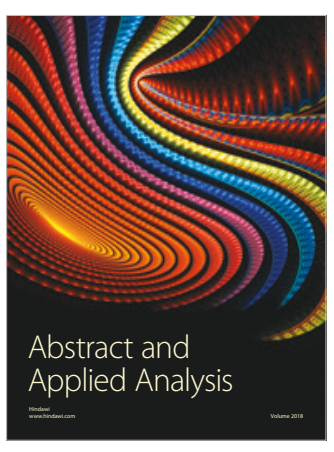

The Scientific

World Journal

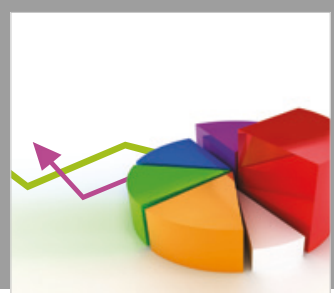

Journal of

Probability and Statistics
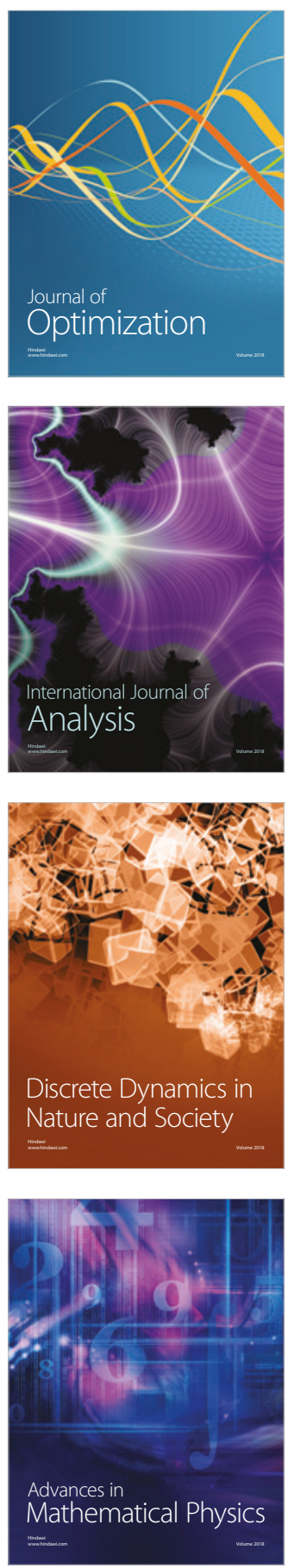